\title{
EVALUATION OF OIL SAMPLES FROM CENTRIFUGAL SEPARATORS
}

\begin{abstract}
Amitabh Jam, S. Biswas, Sadhna Shrivastava and C. R. Jagga
The cleanliness of lubricating oil in plant equipment is of prime concern since particulate contamination can lead to undesirable wear. It is important to be able to measure the size distribution of such particles in order to control contamination levels. This paper describes our recent experience with turbogenerator oil. The presence of emulsified water and particle agglomeration causes serious complications with the analysis of oil samples. The disturbing effect of these factors can be eliminated by treatment of the sample with isopropyl alcohol and heating respectively.
\end{abstract}

Keywords: particulate contamination, particle size distribution, centrifugal separators

\section{Introduction}

The cleanliness of the lubricating oil used in turbogenerators in the power generation industry must meet stringent specifications ${ }^{1}$. The oil is often also used in a servo system which provides generator control. Particles of dirt interact with moving surfaces to produce wear debris and can lead to a chain reaction of wear. The need for cleanliness is generally fulfilled by employing centrifugal separators. These are capable of removing a large portion of particular contamination (in the purifier mode) or water (in the clarifier mode). In this paper we are concerned with particulate removal.

In earlier reports ${ }^{2,3}$ we evolved a methodology for the evaluation of centrifugal separators with regard to particulate removal in simple test situations. Nominally clean oil was dosed with specified particulate contamination. This oil was passed through a separator under test conditions. Samples obtained before and after the separator were compared in terms of particle size distribution. A major complication in measuring such a distribution is the interference by undissolved water. Droplets of water interact optically to produce spurious effects in measurement techniques which employ a light beam. It was possible to restrict the presence of water in the oil to incidental levels since we were concerned with idealized test conditions. The water was dissolved by heating the samples meant for particle counting.

The present work extends the evaluation of centrifugal separators to real-life conditions where, as a result of adverse system conditions, high levels of water might be present in the oil.

Another point of difference with respect to our earlier work is the use of laser-based equipment which employs the phenomenon of light scattering rather than equipment based on the lightblockage principle. This has certain advantages as discussed below. However, each technique is susceptible to the presence of undissolved water and this problem requires careful understanding and handling.

\section{Equipment}

All measurements were made on a Spectrex Laser Particle Counter (model number ILI-I000, serial number 1333). This equipment requires only approximately $200 \mathrm{ml}$ of prepared sample for a measurement. The sample is held in a glass bottle of specified design during measurement. 
The sample is not consumed in any sense. It is available for a modification of sample preparation conditions (in terms of increased dilution, heating or shaking) and repeated measurements if the results so necessitate. In the older equipment the sample flows through a sensor and long piping, and eventually ends up as waste. Thus a large quantity of sample must be available in cases where optimization of sample preparation conditions needs to be carried out. Examples of optimization are given later in this paper.

In the older equipment there are constraints on the permissible viscosity of the sample since this must pass through the sensor at a narrowly specified flow rate. Another problem is frequent blockage of the sensor by large particles. There is also concern related to the cleanliness of the filtered compressed air used to effect flow of sample. In the present equipment the sample remains sealed during measurement. The present equipment is also capable of going down to lower particle sizes although this is not required in the context of the present work.

Clean oil for dilution is obtained from a Hiac Flush Stand which consists of a reservoir from which oil is pumped through a $3 \mu \mathrm{m}$. and then a $0.9 \mu \mathrm{m}$. filter to a tap and a fountain. All glassware used in connection with sample collection, preparation and measurement is washed on this Flush Stand.

\section{Calibration}

Since the use of this equipment is relatively recent, we now present calibration information. We weighed $60 \mathrm{mg}$ of ACFTD (Air Cleaner Fine Test Dust) and dispersed this in $600 \mathrm{ml}$ of Flush Stand oil FS oil). We mixed $100 \mathrm{ml}$ of this stock with $1900 \mathrm{ml} F S$ oil. Finally, we took $100 \mathrm{ml}$ of this diluted stock and mixed it with $100 \mathrm{ml} F S$ oil in a sample bottle and this formed the calibration fluid. At this stage the concentration of ACFTD amounts to $2.5 \mathrm{mg} / \mathrm{l}$. The standard code ISO 4402-1977(E) gives the particle size distribution in ACFTD. The distribution to be expected in the calibration fluid on the basis of this code is shown in Fig 1. This figure also shows measurements of the distribution at the chosen equipment threshold settings. Measurements are shown corresponding to two different dates, the earlier one being the date of calibration. The difference between the two represents the worst-case variation in measurements from one day to another. All values plotted in this work are the average of several measurements taken to ensure reproducibility.

An important detail is heat treatment of the dispersion in oil prior to measurement. In this treatment we heated the sample bottle in an oven until it was warm to the touch. Our experience shows that this is necessary in general. The heating appears to break up agglomerates of particles. An example is given later in connection with an actual sample. Warm water can be used in the ultrasonic bath (which is used after shaking) as an added precaution. Overheating in the oven must be avoided as any charring of the oil causes particle generation. 


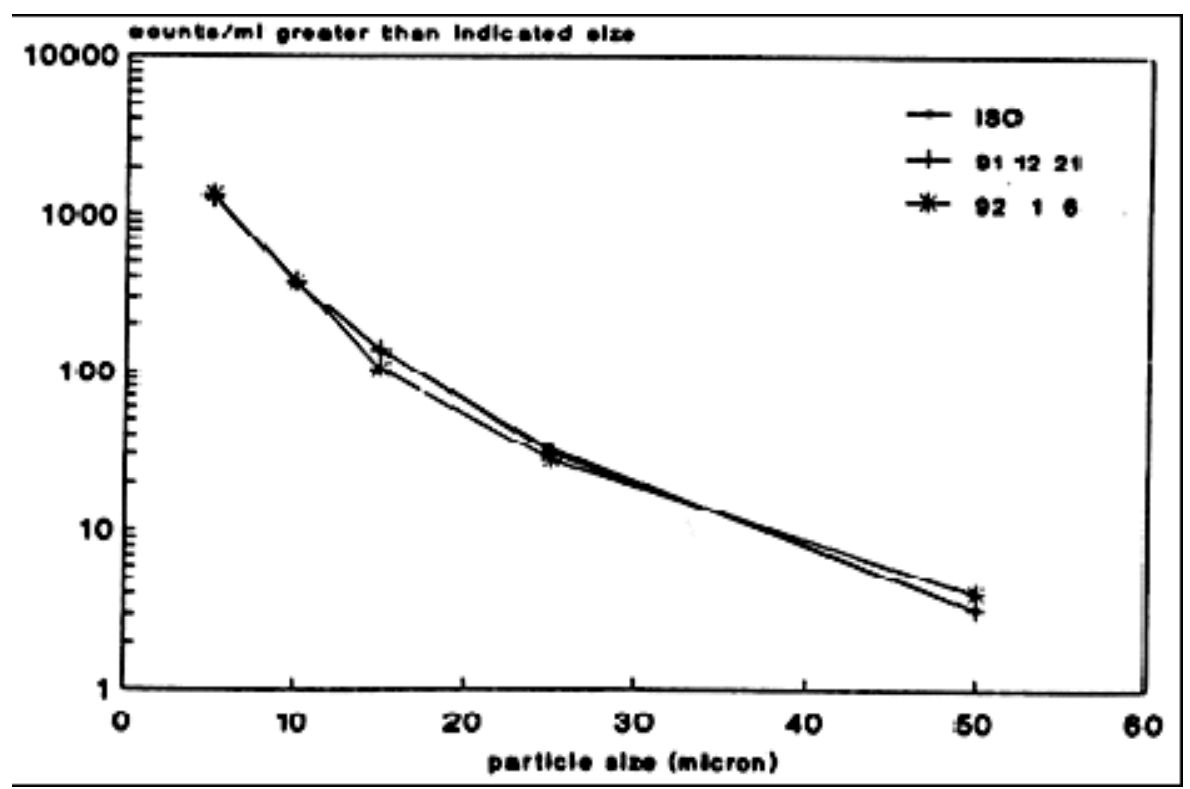

Fig 1 Particle size distribution in fluid containing $2.5 \mathrm{mg} / \mathrm{l}$ ACFTD measured on different dates and the distribution according to ISO code 4402-1977 (E)

\section{Coping with water}

Specific amounts of water were introduced into the oil used to test centrifugal separators in the present work in addition to dosing with particulate contamination. This was done in order to assess particle-removal performance of a separator in the presence of water. There is a concern that in real life large amounts of water may leak into the oil unexpectedly due to system faults. The question then is whether particle removal would be affected.

The presence of water in samples interferes seriously with particle counting. In the past we were only concerned with small amounts of water and heating the sample was adequate to put all the water into solution. In the present study we encountered large concentrations of water even in samples diluted for particle counting. The following experiment illustrates the effect of undissolved water and demonstrates the principle employed in the present work for coping with this.

We took approximately $200 \mathrm{ml}$ of ES oil in a sample bottle and introduced one drop of filtered deionized water into it. One of the curves in Fig 2 shows the measured particle count just after vigorous shaking and ultrasonics (no heating at any stage). A very high count is registered in each of the size ranges. The actual counts are higher since these data are not corrected for the fact that the opacity is $50 \%$, which is higher than that of the calibration fluid (35\%). Further, the counter exhibits saturation effects when the counts registered are very much higher than $1000 / \mathrm{ml}$.

The reason for these counts is that shaking disperses the water into a large number of fine droplets which scatter light and give rise to false counts that are not indicative of particle contamination. This concentration of water is too high to be put into solution by heating. We found that we could reduce the false counts substantially but not eliminate them altogether. We then used the alternative approach now described.

We added isopropyl alcohol (IPA) taken straight from its bottle (supplied by BDH and of General Purpose Reagent' grade) using a new disposable factory cleaned pipette tip for each addition. Figure 2 shows the reduction in counts when $5 \mathrm{ml}$ of $10 \mathrm{ml}$ IPA is added (without heating at any stage). The opacity in each case is also reduced to an appropriate value so that these are true 
counts. We see that the addition of $10 \mathrm{ml}$ is adequate to reduce the counts to near-zero values. Actual samples were treated with IPA during sample preparation. The amount of IPA used in the sample case was based on the data just presented and depended on the likely level of water in each case. We also describe later how we may verify that the effect of water has been annulled in samples bearing particles.

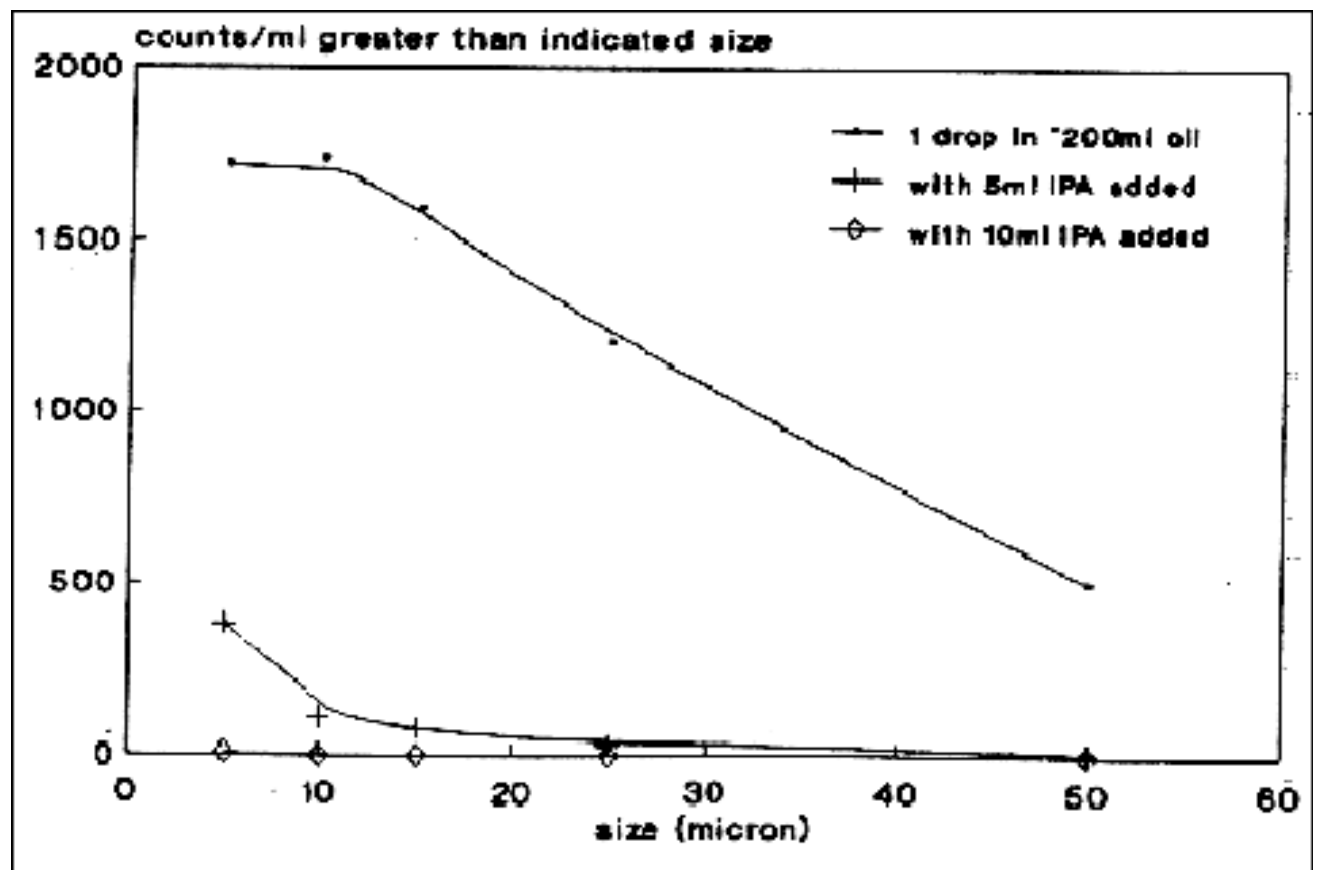

Fig 2 False counts obtained in a bottle of clean Flush Stand oil containing one drop of water and their reduction by treatment with isopropyl alcohol

\section{Results and discussion}

In order to obtain solid contaminant for the dosing of oil meant for separator evaluation, cast iron powder was passed successively through sieves of sizes 53,37 and $25 \mu \mathrm{m}$. This produces powders in the size ranges $37-53 \mu \mathrm{m}, 25-37 \mu \mathrm{m}$ and $<25 \mu \mathrm{m}$. A mixture of equal parts by volume of these powders was used for dosing. The level of dosing was $0.05 \%$ by volume. Dosed oil was passed through separators under test at a flow rate of $6000 \mathrm{l} / \mathrm{h}$ in a single pass. In addition, water was added to a concentration of $1.5 \%$ by volume for the reasons mentioned above.

Figure 3 shows particle size distributions in samples of oil collected after a separator and prepared in various ways. The point labelled 20/200' was obtained on a sample prepared as follows. We poured $130 \mathrm{ml}$ ES oil into a cleaned sample bottle followed by $20 \mathrm{ml}$ IPA and checked by counting that the fluid was clean. We then added $20 \mathrm{ml}$ sample. We then took $30 \mathrm{ml}$ FS oil and used this to rinse out the measuring cylinder used for the sample and to make up the fluid in the sample bottle to $200 \mathrm{ml}$. The counts obtained in the higher size range were too low for any meaningful statistics.

The curve labelled '40/200' was obtained with twice the concentration of sample and IPA (using the sequence $100 \mathrm{ml}$ ES oil, $40 \mathrm{ml}$ IPA, check for cleanliness, $40 \mathrm{ml}$ sample, $20 \mathrm{ml}$ ES oil). This concentration was employed for all later samples collected after separation. The counts corresponding to $5 \mathrm{~m} \mathrm{~m}$ are approximately doubled. This confirms that the effect of water has been annulled. (Measured counts do not scale with dilution when there is undissolved water this fact is explained later.) We now show the effect of warming the sample. 
The curve labelled '40/200 measured warm' refers to the same sample as measured warm (prior heating in the oven and warm ultrasonics). The distribution now has a different shape and represents a decrease in the higher size ranges and an increase in the lower size ones. This is most likely due to the break-up of loose agglomerates of small particles on heating and is therefore a desirable effect. We have adopted our procedure of making measurements on warm samples as a standard routine and this is not specifically mentioned hereafter.

Figure 4 shows data for a dosed sample. The concentrations used in this case are $5 \mathrm{ml}$ sample and $10 \mathrm{ml} \mathrm{IPA} \mathrm{mixed} \mathrm{with} 185 \mathrm{ml}$ ES oil. This is the concentration used for all dosed samples.

The curve labelled ' $5 / 200$ ' shows the raw data. As a test we doubled the dilution by discarding $100 \mathrm{ml}$ from the sample bottle and replacing it with $100 \mathrm{ml}$ clean ES oil. The curve labelled 'doubled dilution' shows the resultant distribution. The counts are halved. If there was a certain fraction of undissolved water in the former case, doubling the dilution would result in lowering of this fraction by a factor greater than two (the fresh ES oil would dissolve some water). The component of counts due to free water would not therefore scale by a factor of two and neither would the total counts. Thus a true scaling of counts is an assurance that all water has been dissolved.

\section{Conclusions}

The addition of isopropyl alcohol when preparing samples is an effective method of dissolving water which would otherwise obscure the true particle count.

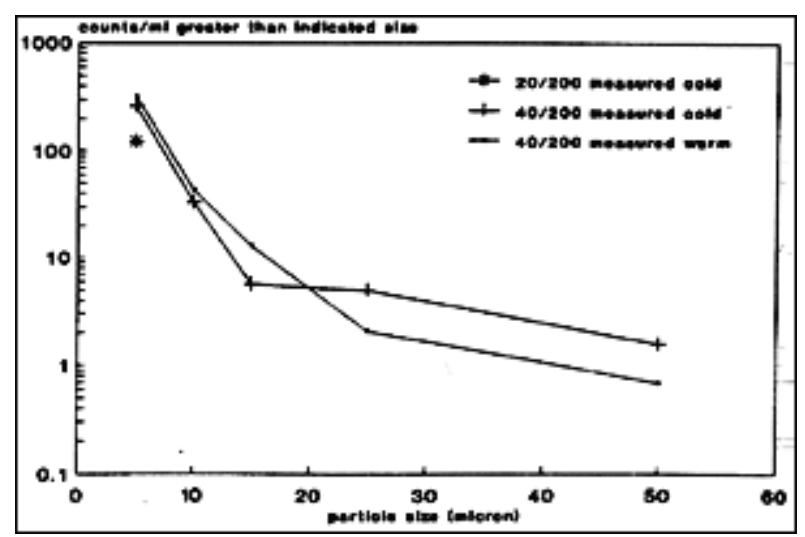

Fig 3 Particle size distribution in $200 \mathrm{ml}$ fluid containing $20 \mathrm{ml}$ or $40 \mathrm{ml}$ centrifuged oil as measured cold or warm 


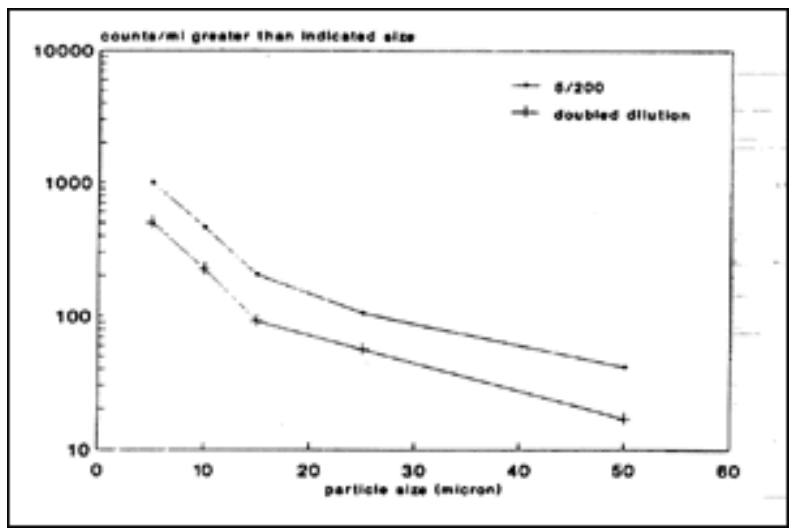

Fig 4 Particle size distribution in $200 \mathrm{ml}$ fluid containing $5 \mathrm{ml}$ dosed oil and at double this dilution

It is also necessary to heat samples prepared for measurement in order to obtain distributions that otherwise appear to be distorted by particle agglomeration.

\section{Acknowledgements}

We record our appreciation of the assistance rendered by M. S. Rawat and Lars Theustad in the laboratory.

\section{References}

1. Cleanliness Requirements of Parts Used in Hydraulic Systems, NAS 1638, January 1964, Aerospace Industries Association of America, Inc. NAS class 5 is applicable to the lube oil system of stationary powergeneration turbines

2. Jagga C.R. and Amltabh Jam, Evolving a Procedure for Evaluation of Centrifugal Separators Using a Particle Counter, Consultancy Report, 1985, IIT Delhi

3. Amitabh Jam, Blswas S, and Sadhna Shrivastava, Contaminant Analysis for Centrifugal Separator Evaluation, Consultancy

Report, 1988, IIT Delhi 